\title{
Análisis del diseño y material de un convertidor de par de una transmisión automática sobre el desempeño de torque y potencia de un automóvil liviano.
}

\begin{abstract}
Analysis of the design and material of a torque converter automatic transmission on the performance of torque and power of a car light.
\end{abstract}

Paúl Montúfar Paz. ${ }^{1}$, Víctor David Bravo Morocho. ${ }^{2}$, Edison Marcelo Castillo Cardenas. ${ }^{3}$, Edgar Fabián Sanchez Carrión. ${ }^{4}$ \& Edison Patricio Abarca Perez. ${ }^{5}$

\begin{abstract}
.
DOI: $\underline{\text { https://doi.org/10.33262/cienciadigital.v2i3.176 }}$

The torque converter into the automatic drivetrain has been quite important as well as the hydraulic clutches due to the capacity of amplifying the torque highly accurate over $98 \%$ of low RPM, working as attachment of 1:1in high RPM, to get a better efficiency and low gas intake for saving the environment. Because of its design and fuction, the torque converter engages and disengages automatically the power of the engine to the gear, focusing on the engine RPM. With the powertrain on, at an idling speed, the power is weak due to the lack of fluid flow; However, when speeding up, the powertrain gets stronger through the torque converter job. This research aims on the torque converter features based on the main variables. The first one was a test on the dynamometer for finding the torque and power curve, and the second one, an intake test. This study was made at CCICEV in order to understand how the torque converter works in automatic vehicles. A didactic board was built for seeing the torque graphics, power and slippery of the converter in order to verify possible damage in advance. This research was pretty important for the Automotive Engineering students from the Mechanics Faculty.
\end{abstract}

Keywords: Automatic Transmission, Torque Converter, Rescuer, Consumption.

\footnotetext{
${ }^{1}$ Escuela Superior Politécnica de Chimborazo, Chimborazo, Ecuador, pamp6010@ @otmail.com.

${ }^{2}$ Escuela Superior Politécnica de Chimborazo, Chimborazo, Ecuador, victor.bravo@espoch.edu.ec.

${ }^{3}$ Escuela Superior Politécnica de Chimborazo, Chimborazo, Ecuador, edison.castillo@espoch.edu.ec

${ }^{4}$ Escuela Superior Politécnica de Chimborazo, Chimborazo, Ecuador, esanchez_c@espoch.edu.ec.

${ }^{5}$ Escuela Superior Politécnica de Chimborazo, Chimborazo, Ecuador, edison.abarca@ espoch.edu.ec.
} 


\section{Resumen.}

El convertidor de par en el tren de transmisión automático ha sido muy importante, así como los embragues hidráulicos debido a la capacidad de amplificar el par de alta precisión más del 98\% de bajas RPM, trabajando como accesorio de 1: 1 a altas RPM, para obtener una mejor eficiencia y baja ingesta de gas para salvar el medio ambiente. Debido a su diseño y reducción, el convertidor de par activa y desactiva automáticamente la potencia del motor para el engranaje, centrándose en las RPM del motor. Con el tren motriz encendido, a velocidad de ralentí, la potencia es débil debido a la falta de flujo de fluido; Sin embargo, cuando se acelera, el tren de potencia se fortalece a través del trabajo del convertidor de par. Esta investigación tiene como objetivo las características del convertidor de par basadas en las variables principales. El primero fue una prueba en el dinamómetro para encontrar el torque y la curva de potencia, y el segundo, una prueba de admisión. Este estudio se realizó en CCICEV para comprender cómo funciona el convertidor de par en vehículos automáticos. Se construyó un tablero didáctico para ver los gráficos de torque, potencia y deslizamiento del convertidor para verificar posibles daños por adelantado. Esta investigación fue muy importante para los estudiantes de Ingeniería Automotriz de la Facultad de Mecánica.

Palabras Claves: Transmisión Automática, Convertidor De Par, Rescatador, Consumo.

\section{Introducción .}

En el proyecto de titulación presentado se abordará los principales conceptos, diagramas y análisis sobre la caracterización del convertidor de par de la transmisión automática a diferentes cargas del motor. En nuestra condición geográfica existe un ciclo de conducción particular, del cual es necesario realizar el estudio y análisis para determinar el comportamiento del convertidor de par realizando pruebas dinamométricas para analizar si el convertidor es el idóneo para el tipo de motor de combustión interna en función al torque, potencia y consumo, debido a que es un componente que está sujeto a muchas mejoras.

Existen investigadores que han aportado diferentes estudios sobre el comportamiento del convertidor, muchos de estos estudios no garantizan resultados reales y exactos. Este elemento está diseñado en base al torque que necesita vehículo para vencer su propia inercia y a la condición de vía que va a ser sometido. Se espera que esta investigación permita una mejor aplicación en el área de la enseñanza en la Escuela de Ingeniería Automotriz.

\section{Importancia del problema.}

Debido a las mejores prestaciones con las que cuenta un vehículo con transmisión automática día a día se va incrementando el parque automotor con este tipo de tren de 
potencia, sin embargo, en nuestro país existe cierta resistencia por inclinarse por este tipo de vehículos al momento de la compra debido a:

- Alto costo de mantenimiento.

- Desconocimiento de su operación.

- Costo de los repuestos.

Parte de estos motivos es posible subsanarlo con un mejor entendimiento del funcionamiento de este tipo de transmisión el mismo que busca proporcionar mayor comodidad de conducción y un rango de operación en el cual se optimicen las prestaciones tales como Torque potencia y consumo.

La transmisión automática al ser un conjunto de componentes mecánicos con un control hidráulico determinado por componentes electrónicos requiere de un conocimiento integral de mecánica hidráulica y electrónica para su diagnóstico, reparación y en fases superiores diseño. Todos estos aspectos si bien requieren de conocimiento y experiencia dejan de ser complejos en el momento que se logra desarrollar la capacidad de entender su operación, por estos motivos el sector automotriz nacional requiere de una sociabilización de los aspectos fundamentales a fin de que los técnicos automotrices puedan operar las herramientas de diagnóstico y reparación para el desarrollo de este tipo de transmisiones.

El convertidor de par al ser uno de los componentes principales de la transmisión está sujeto a una serie de aspectos de construcción y diseño que logra el consumo de combustible pues reducen las fuerzas de fricción durante la transmisión de movimiento, de modo que el conocimiento de sus parámetros de operación y la ingeniería involucrada en el mismo permitirán dirigir futuros estudios a la optimización de este componente para proporcionar mejores características de transmisión de movimiento en el vehículo.

\section{Metodologia .}

Convertidor de par

Los accionamientos hidrocinéticos implican la transferencia de energía a través de la "energía cinética" o la velocidad de un fluido. En tales dispositivos, un elemento impulsor crea la energía cinética del flujo y un elemento de turbina recupera la energía que produce una salida de par. Hay dos tipos principales de dispositivos hidrocinéticos: acoplamiento de fluido y convertidor de fluido. Ambas familias proporcionan un ajuste automático de la relación (velocidad de entrada para una velocidad y carga de salida dada) y una capacidad de relación infinita que los hace muy apropiados como un "dispositivo de arranque".

El término convertidor de par se usa aquí para describir el acoplamiento de convertidor como el más frecuentemente usado en aplicaciones automotrices. Esto también se conoce como un convertidor de Trilok. Se llama así porque en una parte de su rango de funcionamiento da una multiplicación de par (que actúa como un convertidor) y en el resto se comporta como un acoplamiento con una relación de par 1: 1 (VAUGHAN, 2002). 
Figura 1 Esquema del convertidor de par

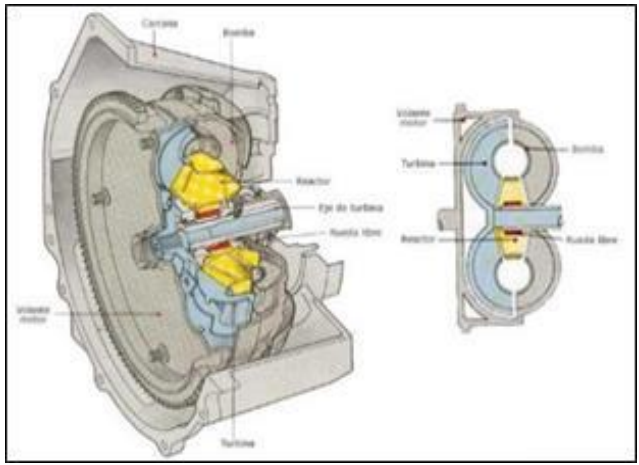

Fuente:http://www.aficionadosalamecanica.net/caja-cambios3.htm

\section{Funcionamiento del convertidor de par.}

Al girar la bomba accionada directamente por el movimiento del cigüeñal, el aceite se impulsa desde la rueda de bomba hasta la rueda turbina. A la salida de ésta el aceite tropieza con los alabes del reactor que tienen una curvatura opuesta a los de las ruedas de bomba y turbina (Transpart, 2014).

Esta corriente de aceite empuja al reactor en un giro desentido contrario al de la bomba y la turbina. Como el reactor no puede realizar ese giro ya que está retenido por la rueda libre, el aceite se frena y el empuje se transmite a través del aceite sobre la bomba. De esta forma mientras exista diferencia de velocidad de giro entre la bomba y la turbina el momento de giro (par) será mayor en la turbina que en la bomba. El par cedido por la turbina será pues la suma del transmitido por la bomba a través del aceite y del par adicional que se produce por reacción desde el reactor sobre la bomba y que a su vez es transmitido de nuevo sobre la turbina. Cuanto mayor sea la diferencia de giro entre turbina y bomba mayor será la diferencia de par entre la entrada y la salida del convertidor, llegando a ser a la salida hasta tres veces superior.

Figura 2: Funcionamiento del convertidor de par

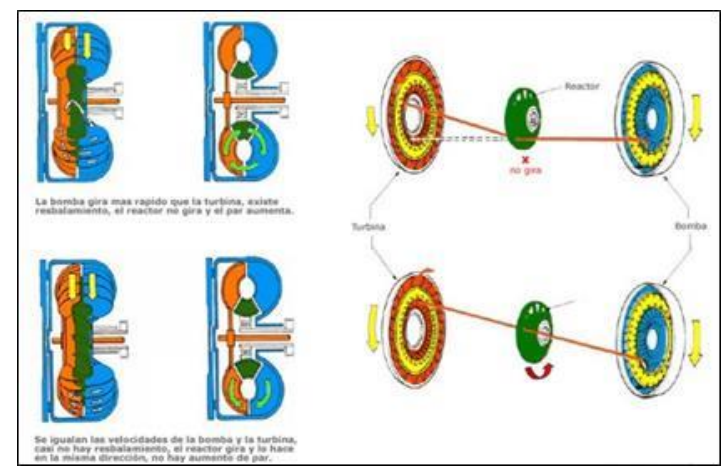

Fuente: http://www.transpart.com/7.html 
Conforme disminuye la diferencia de velocidad va disminuyendo la desviación de la corriente de aceite y por lo tanto el empuje adicional sobre la turbina con lo que la relación de par entre salida y entrada va disminuyendo progresivamente.

Cuando las velocidades de giro de turbina e impulsor se igualan, el reactor gira incluso en su mismo sentido sin producirse ningún empuje adicional de forma que la transmisión de par no se ve aumentada comportándose el convertidor como un embrague hidráulico convencional. A esta situación se le llama "punto de embrague". (ATSG, 2004)

El convertidor de par funciona en dos etapas. En la primera etapa el convertidor de par es capaz de multiplicar el par motor que recibe.

En la segunda etapa, a medida que se va subiendo las RPM, el convertidor de par reduce el factor multiplicador de par motor hasta quedar en una relación de 1:1, donde se transmite aproximadamente el $98 \%$ del giro del motor, existiendo un $2 \%$ que se pierde por resbalamiento.

\section{Partes del convertidor de par.}

El convertidor de par consta de tres partes fundamentales que son:

\section{Impulsor o bomba Estator o reactor Turbina}

Figura 3: Partes del convertidor de par

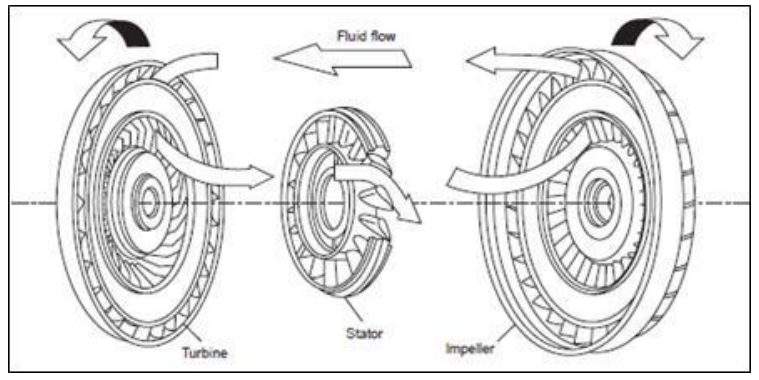

Fuente: (VAUGHAN, 2002)

\section{Impulsor.}

Órgano a través del cual penetra la potencia en el convertidor de par. Corrientemente, el impulsor recibe el nombre de bomba, lo que técnicamente es exacto. No obstante, se recomienda utilizar el nombre de impulsor, para evitar la confusión con las bombas de presión y otros tipos de bombas.

\section{Estator.}

El convertidor de par incluye un elemento que mejora las condiciones de funcionamiento en la circulación del aceite, se trata del estator. Tiene como misión redirigir el aceite ocupado por la turbina y entregarlo al impulsor, cambia de dirección el flujo de aceite, esto permite aumentar el impulso del aceite. Dentro del estator se encuentra un cojinete de un solo sentido, lo que permite que este solo gire en un determinado sentido. El estator 
se usa para redirigir el flujo de la turbina de regreso hacia la parte de la bomba, para completar el flujo de aceite. Está montado sobre un mecanismo de rueda libre que le permite desplazarse libremente cuando los elementos del convertidor giran a una velocidad aproximadamente igual (VAUGHAN,

2002).

\section{Turbina.}

El elemento conducido se llama turbina, y va acoplada a la caja de cambios. La parte de la bomba del convertidor de par dirige aceite presurizado contra la turbina para hacerla girar. La turbina está conectada a una flecha, para transferirle potencia a la transmisión. Tiene como misión recibir el aceite enviado por el impulsor. La turbina gira en conjunto con el eje de salida ya que estos están unidos en un mismo eje.

\section{Ventajas del convertidor de par.}

- Las ventajas del convertidor de par son:

- Variación continúa en el par y la velocidad sin control externo

- Aislamiento de la vibración Absorción de la carga de choque Mantenimiento bajo

- Operación virtualmente libre del desgaste.

\section{Mejora del rendimiento del convertidor de par.}

Para aumentar el rendimiento de funcionamiento, se construye interiormente al impulsor y a la turbina la llamada corona directriz partida. Esta sirve para suavizar y uniformizar el movimiento del líquido entre ambos miembros, impulsor y turbina, dado que en el centro del movimiento vertical se establece una turbulencia cuyo resultado es una pérdida de rendimiento.

Figura 4: Convertidor de par de tres elementos

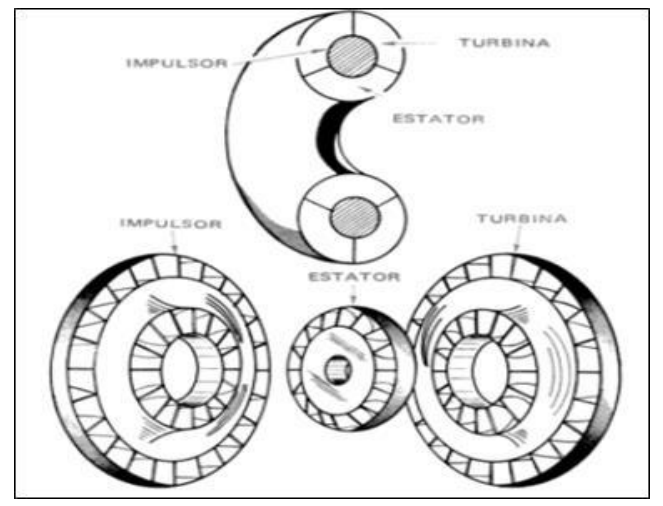

Fuente: General motors

Otra característica constructiva es la configuración de los álabes del impulsor y dela turbina que se representa en figura. A los álabes del impulsor se les da una forma curvada hacia atrás para aumentar la aceleración y la energía del aceite que lo abandona.

\section{Refrigeración del convertidor de par.}


Por el trabajo que realiza el convertidor de par se hace necesario disponer de algún medio de refrigerar el líquido para que este no se recaliente demasiado. Se utilizan dos sistemas de refrigeración: por aire y por agua.

En ambos tipos, el aceite de alimentación a baja presión procedente de la bomba delantera del cambio y del regulador de presión entra y sale continuamente del convertidor, regresando el caudal de salida al colector de aceite. En los sistemas de refrigeración por agua, utilizados en los motores de mayor potencia, simplemente se hace pasar el aceite por un depósito de refrigeración situado debajo del radiador de agua o junto a este.

Figura 5: Refrigeración del convertidor de par.

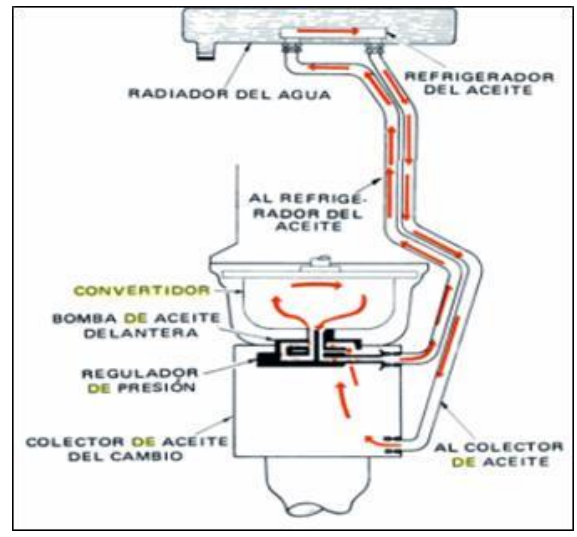

Fuente: Ford Motor Co.

\section{Estrategia de control del convertidor de par.}

El concepto, características y diseño de los tres componentes del sistema de un convertidor de par - el embrague de bloqueo, el amortiguador y el circuito hidráulico - son el resultado de la estrategia de control requerida para el embrague de bloqueo.

La estrategia de control es el enlace central de los elementos de hardware; lo que determina los requisitos para diferentes rangos de operación y, por lo tanto, el diseño de los componentes individuales. La estrategia de control de bloqueo apropiada se define individualmente para cada aplicación del vehículo considerando la funcionalidad del vehículo y los objetivos para la economía de combustible, el rendimiento, la conducción y la comodidad del conductor (MIDDELMANN,

2006). UNITS 
Figura 6: Sistema total de convertidor de par.

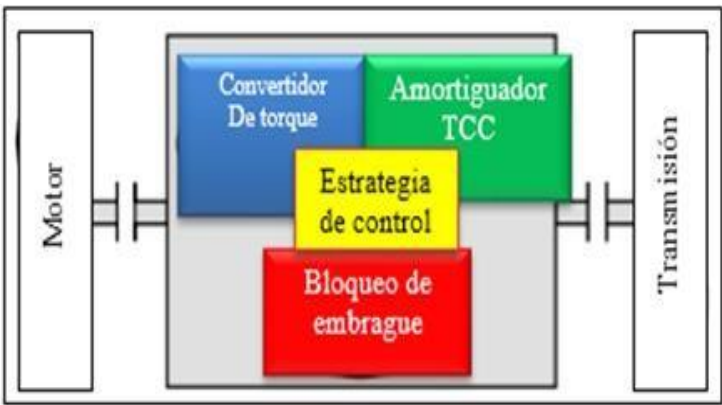

Fuente: (MIDDELMANN, 2006).

\section{Mejora de la eficiencia del convertidor de par.}

La mejora de la eficiencia de un convertidor de par se puede lograr mediante el cambio de la geometría de las aspas. Esto se puede comprobar mediante software de simulación de flujo de fluidos.

Figura 7: Modelación convertidor de par

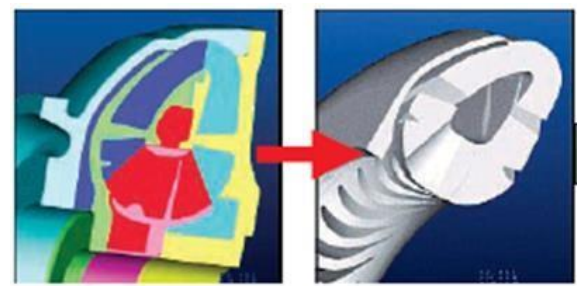

Fuente: (KOMATSU, 2004)

La longitud de la hoja ajustada, la posición de la curvatura de la hoja, ovalidad, etc., son otros factores que afectan en la eficiencia. (KOMATSU, 2004) SOME COMMON MISTAKES.

\section{Diseño metodológico.}

Curvas características del convertidor de par

El convertidor de par de Chrysler Corp. tiene la siguiente curva característica:

Grafica 1: Curva característica del convertidor de par Chrysler

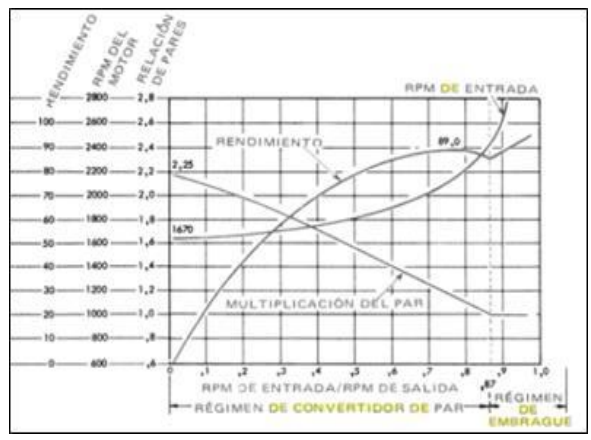

Fuente: Chrysler Corp. 


\section{Relación de velocidades.}

La relación o cociente de velocidades, es el número de vueltas que da la turbina por cada vuelta que da el impulsor. Es también una medida de rendimiento del embrague o del convertidor y se expresa en porcentaje. Por ejemplo, si el impulsor gira a $1000 \mathrm{rpm}$ y la turbina a $900 \mathrm{rpm}$, la relación de velocidades es de $90 \%$.

Relación de velocidad $=$ rpm de la turbina $/ \mathrm{rpm}$ del impulsor .

En el preciso momento en que el automóvil comienza a moverse, hay un momento en que el impulsor está girando, pero la turbina no ha empezado a moverse: la relación de velocidades es cero. Mientras dura esta situación, los movimientos giratorio y vertical del líquido se encuentran en los estados siguientes:

Como la turbina esta quieta, el movimiento vertical se efectúa a través de la turbina sin resistencias, produciéndose una circulación transversal masiva entre los dos miembros

Figura 8: Relación de velocidades nula

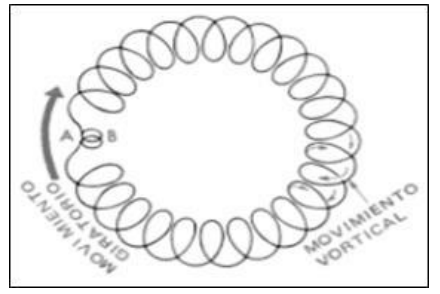

Fuente: Ford Motor Co.

\section{Capacidad de los convertidores.}

Cuando se habla de los convertidores es importante mencionar otra característica referente a su comportamiento. Todo mecanismo de transmisión de potencia por engranajes admite un par de cualquier valor hasta llegar su punto de resbalamiento; sin embargo, los convertidores admiten un par de valor máximo dado y no más. Por ejemplo, a medida que el acelerador se abre y el par motor comienza a elevarse, el motor puede aumentar de velocidad solo hasta el punto en que el par motor alcanza el par máximo admitido por el convertidor. Cuando este alcanza, las rpm del motor se estabilizan y el convertidor resbala. Como velocidad de pérdida del convertidor (prueba de calado).

La turbina inmóvil ofrece también resistencia al movimiento giratorio del líquido y el movimiento de este tampoco lo favorece.

El efecto de aceite que incide sobre la turbina está determinado por la intensidad relativa de ambos movimientos del aceite. En la figura 2-4 se representa el impacto y su resultado mediante un diagrama de vectores. En el mismo se representan ambos movimientos, giratorio y vortical, el hecho evidente de que en el momento del impacto no puede haber movimiento en dos direcciones a la vez. La dirección del aceite que resulte del impacto de ambos movimientos del líquido seguirá un determinado ángulo intermedio, resultante 
de las acciones giratorias y verticales, según determine la relación de velocidades o condiciones de marcha del vehículo.

Figura 9: Movimiento giratorio y vortical

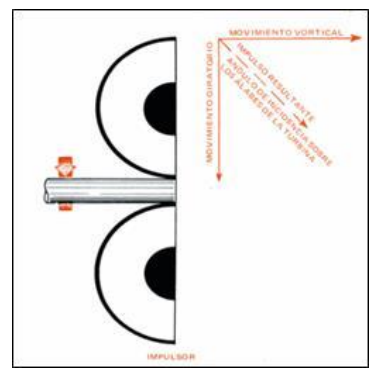

Fuente: Ford Motor Co.

Con esto, ha de resultar evidente que el convertidor desempeña el papel adicional de regular las rpm del motor $\mathrm{y}$, en esencia, debe ser cuidadosamente diseñado para aprovechar el par útil cedido por el motor en las condiciones de rendimiento máximo (convertidor y motor deben formar un conjunto equilibrado).

Grafica2: Par vs velocidad de rotación

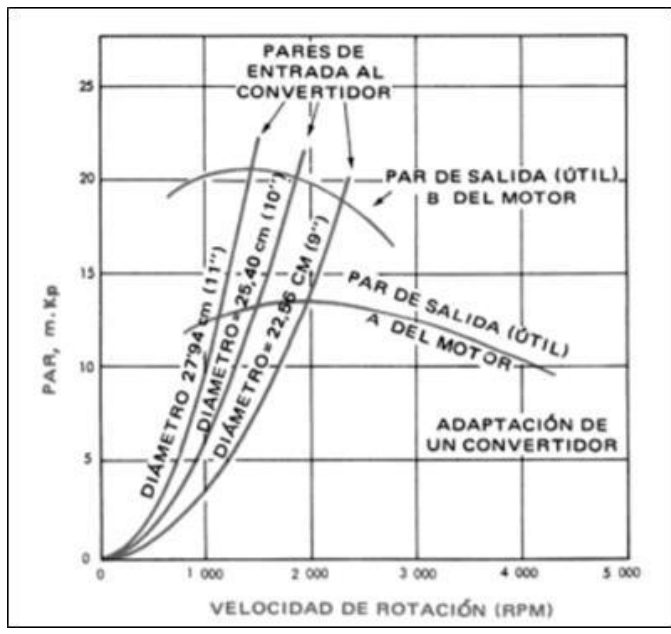

Fuente: Ford Motor Co.

Dicho de otra forma, el régimen del motor está regulado, en virtud de la constitución del convertidor, por la carga que impone el impulsor sobre el motor al tratar aquel de accionar la turbina. Naturalmente, a medida que el vehículo cobra impulso y aumenta la velocidad de la turbina, disminuye el par motor necesario para accionar el impulsor y la velocidad del motor se eleva uniformemente.

Cuando en algún caso particular se utilice un convertidor demasiado pequeño, el motor funcionara a un régimen de revoluciones más elevado que el necesario antes de que el convertidor puede trasmitir el par máximo. En aquellos casos en que se utilice 
un convertidor demasiado grande, el motor no poseerá capacidad para accionar al impulsor a una velocidad que permita a este funcionar a la máxima potencia. En ambos extremos resultan sendas situaciones indeseables, tanto por sobre revolucionado como por infra revolucionado. La práctica corriente es acoplar motor y convertidor a una velocidad de perdida tal que el motor sea capaz de alcanzar su par máximo. De lo que precede resulta evidente que el técnico de servicio no debe alterar las condiciones del acoplamiento motor- convertidor calculadas por el fabricante.

\section{Especificaciones del vehículo.}

El vehículo Daewoo Espero modelo 1995 cuenta con las siguientes especificaciones:

Tabla 1: Especificaciones técnicas Daewoo Espero

\begin{tabular}{ll}
\hline Marca & Daewoo \\
\hline Modelo & Espero \\
Motor & $200 \mathrm{cc}$ \\
Transmission & Automatic \\
Potencia maxima & $105 \quad \mathrm{CV} / \quad 5000$ \\
Par máximo & RPM \\
Año de fabricacion & $169 \mathrm{Nm} / \mathrm{2}$ 2800 \\
Longitud & 1995 \\
Ancho & $4615 \mathrm{~mm}$ \\
Altura & $1718 \mathrm{~mm}$ \\
Distancia entre ejes & $1388 \mathrm{~mm}$ \\
Numero de cuadros & $2620 \mathrm{~mm}$ \\
Combustible & 4 \\
Consumo de combustible urbano & \\
Consume de combustible extraurbano & $\mathrm{Gasolina}$ \\
Consume de combustible combinado & $12,31 / 100 \mathrm{~km}$ \\
\hline
\end{tabular}

Realizado por: Autores.

Fuente: ficha tecnica Daewoo Espero. 


\section{Diseño de experimentos}

Para el experimento se toman en cuenta los siguientes parámetros:

\section{Ecuación de la velocidad vs frecuencia}

La ecuación utilizada para las RPM es la siguiente:

$\mathrm{Y}=0,1787 \mathrm{x}+47,557 \quad$ Eje $\mathrm{Y}=$ frecuencia $(\mathrm{F})$

$\mathrm{F}=0,1787 \mathrm{RPM}+47,557 \quad$ Eje $\mathrm{X}=$ revoluciones

$(\mathrm{RPM})$

La ecuacion final es:

$\mathrm{RPM}=\frac{F-47,557}{0,1787}$

\section{Ecuaciones fundamentales}

Para realizar los cálculos en Excel, se utiliza las siguientes formulas:

Para la relación de velocidades

$\mathrm{V}=\frac{n t}{n b}$

Donde:

$\mathrm{nt}=\mathrm{rpm}$ de la turbina

$\mathrm{nb}=\mathrm{rpm}$ de la bomba (par motor)

para el rendimiento:

$\mathrm{n}=\frac{P t}{P b}$

donde:

$\mathrm{Pt}=$ potencia de la turbina

$\mathrm{Pb}=$ potencia de la bomba (potencia del motor)

Para el resbalamiento:

$S=100 x(1-V)$

Donde:

$\mathrm{V}=$ relacion de velocidades 
Para la relacion de par:

$\mathrm{U}=\frac{M t}{M b}$

Mt: par de la turbina

Mb: par de la bomba

Para el rendimiento

$n=u x v$

\section{Graficas de torque y potencia hallados en CCICEV}

Grafica 3: Curva Potencia vs RPM Fuente: CCICEV

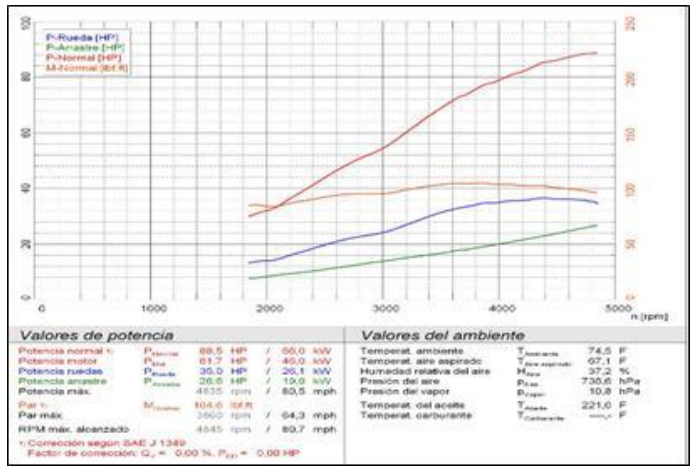

Realizado por: Autores.

\section{Consumo de combustible}

Según la ficha técnica del vehículo Daewoo Espero, el consumo de combustible combinado es de $5.8 \mathrm{lt}$ por cada $100 \mathrm{~km}$.

Tabla 2: Ensayo de consumo

\begin{tabular}{ccccc}
\hline \multicolumn{1}{c}{ Tramos } & Ciclo & Inicio & Final(L) & Distancia(Km) \\
\hline A & IM 240 & 5 & 4,725 & 3,2 \\
B & IM 240 & 5 & 4,73 & 3,2 \\
C & IM 240 & 5 & 4,73 & 3,2 \\
Rendimiento Tramo A(km/gal) & 44,06 & & \\
Rendimiento Tramo B(km/gal) & 44,86 & & \\
Rendimiento Tramo C(km/gal) & 44,86 & & \\
Rendimiento Total(km/gal) & $44-59$ &
\end{tabular}

Fuente: CCICEV 


\section{Resultados.}

Graficas de las curvas principales del convertidor de par del vehículo daewoo espero.

A continuación se muestra la curva de resbalamiento del convertidor de par.

Grafico 4: Resbalamiento.

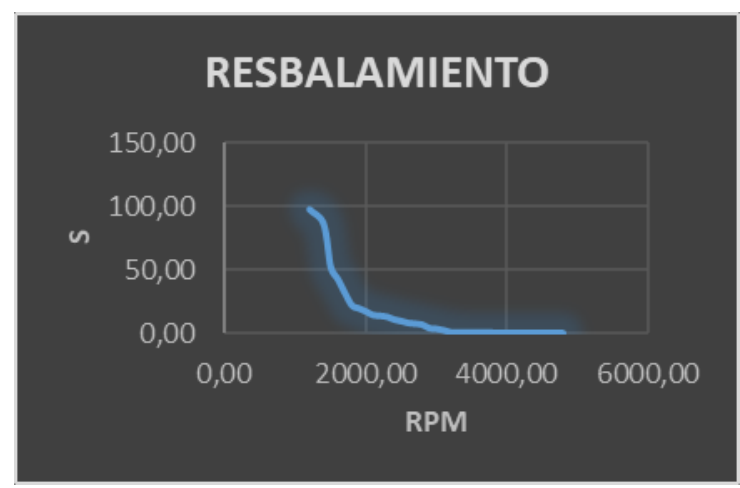

Fuente: Autores.

En la figura se observa el máximo resbalamiento cuando el vehículo ha logrado vencer su inercia llegando a un valor del 98\% a 1200 RPM, posteriormente el resbalamiento disminuye a medida que las RPM aumentan.

Grafica 5: Relación de velocidades.

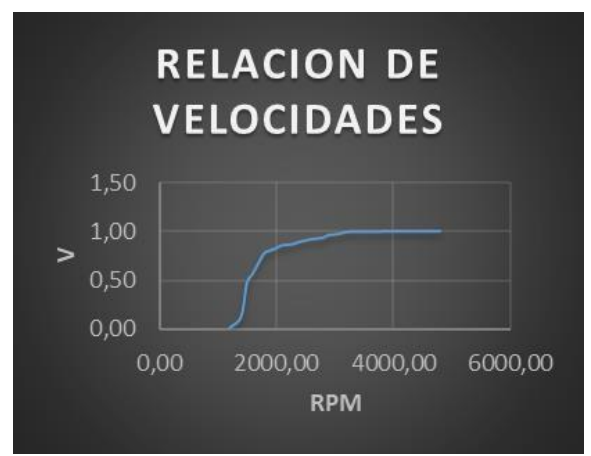

Fuente: Autores.

En la figura se observa que la relación de velocidades es directamente proporcional a las RPM del motor, logrando entender que la fase de acoplamiento entre la turbina y el impulsor se da las 3200 RPM. 
Grafica 6: Relación de torque.

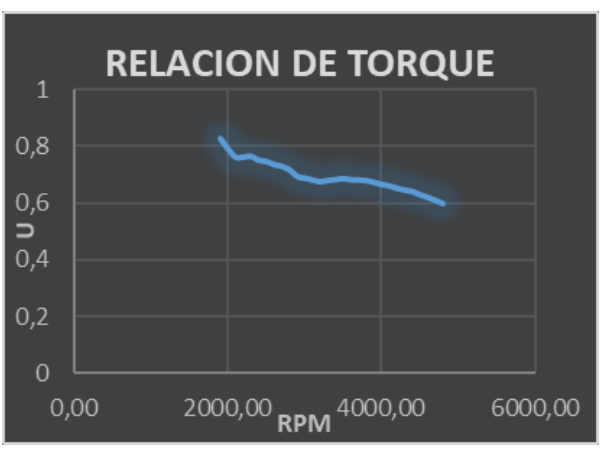

Fuente: Autores.

Se puede observar que el máximo torque del convertidor de par se da a bajas RPM, con un valor de relación de torque del $88 \%$ a 1800 RPM, existen pre-acoplamientos en función de la aceleración del conductor o en base a la geografía de la carretera.

Grafica 7: Potencia vs RPM .

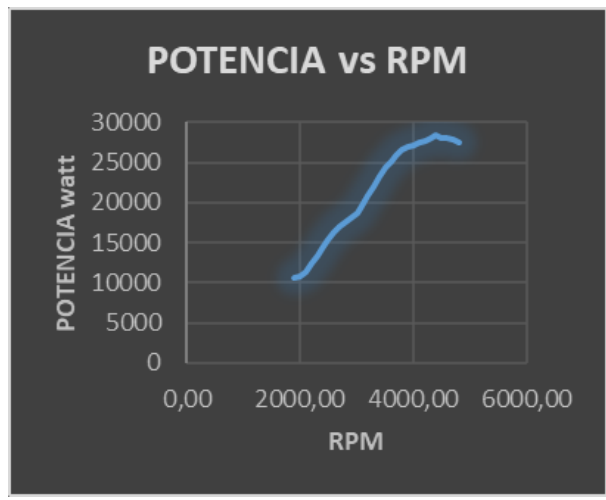

Fuente: Autores.

En la siguiente figura se puede observar que a partir de la etapa de acoplamiento la potencia aumenta conforme las rpm del mot or hacienden, con valores máximos de potencia 27408,59017 watt a $4800 \mathrm{rpm}$.

Grafica 8: Rendimiento

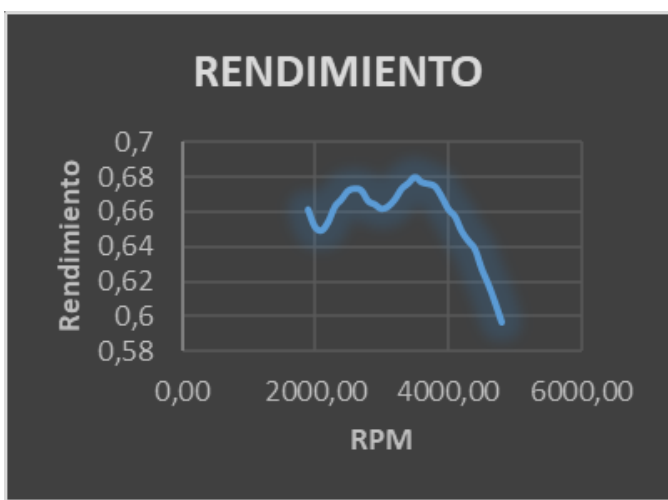

Fuente: Autores. 
En la siguiente figura se muestra que el máximo rendimiento se da antes de la etapa de acoplamiento entre el impulsor y la turbina con valores del 68\% a 3500 RPM, observando también que existen picos debido al pre-acoplamiento del convertidor de par.

\section{Consumo de combustible.}

Según la ficha técnica del vehículo Daewoo Espero, el consumo de combustible combinado es de $5.8 \mathrm{lt}$ por cada $100 \mathrm{~km}$.

El consumo obtenido en los ensayos es el siguiente:

El consumo $E=44,86 \frac{\mathrm{km}}{\mathrm{gal}}$

Dando como resultado un consumo de

$E=44,86 \frac{K M}{g a} \times 1 \frac{g a l}{3,78 l t}=12,12 \frac{\mathrm{km}}{\mathrm{lt}}$

Realizando una regla de 3 con los datos obtenidos, se llega a tener un resultado de consume $E=8,25 \mathrm{lt}$ por cada $100 \mathrm{~km}$.

El resultado final nos da un consume de 2,45 lt superior.

\section{Conclusiones .}

- Se obtuvo las gráficas de torque normal y potencia normal del convertidor, con valores máximos de $\mathrm{Tn}=135,74 \mathrm{Nm}$ a $3545 \mathrm{RPM}$; Pot.N=88,5Hp a 4835RPM, mediante el cual existe un perdida por resbalamiento del convertidor de par.

- Se determine mediante los valores obtenidos en el banco dinamométrico, que el convertidor de par tiene un rendimiento del 63\% a 1900 RPM, existiendo una pérdida del $35 \%$ por resbalamiento y tiene que ser sustituida.

- Se obtuvo las curvas de torque-potencia en el motor y hacia las ruedas, a partir de esta se calculó las curvas principales de funcionamiento es del 63\% a 1900 RPM, con un resbalamiento máximo del 19,89\% RPM, un torque máximo de $66,30 \mathrm{Nm}$ a 1900 RPM y una potencia máxima de 38 Hp a 28340 RPM.

- Se verifico el consumo de combustible mediante un ensayo, el cual arrojo un resultado de que existe un consume de 1 litro por cada $12,12 \mathrm{Km}$, por lo tanto consume 8,25 litros por cada $100 \mathrm{~km}$ recorridos, dando un resultado mayor al estimado por el fabricante que declara en su ficha técnica un consume de 5,8 litros por cada $100 \mathrm{Km}$ recorridos, teniendo un desfase de 2,45 litros, que son ocasionados por la antigüedad del vehículo, y el resbalamiento que se produce en el convertidor de par. 


\section{Referencias bibliográficas.}

Automatic transmission service group. ATSG. ( En linea) Miami- USA, 2004 (consultado el 10 de Agosto de 2017). Disponible en: http://www.atsg.us/atsg/

Automotive transmissions fundamentals HARALD, naunheimer, selection, desing and application. Berlin alemania, 2010, pp 100-114

Manual de transmisiones automaticas ,General motors, corp,. ( en linea) (consultado el 9 de septiembre de 2017). Disponible en https://www.gm.com/..

Prediccion of torque converter characteristics KOMATSU KOMATSU.. ( en linea) Japon, 2004. Disponible en: https://home.komatsu/en/company/tech. innovation/report/pdf/154-02 E.pdf

Tractors y motores Arnal atares, Pedro V agricolas. Madrid-España. Ediciones Mundi Prensa, 2000. Pp. 2-4

Transmission and driveline VAUGHAN, Nick.- An Introduction to modern vehicle desing . Oxford, 2002. Reed Educational and Professional Publisging Ltd, 2002. ISBN 075065044 3. PP 260-280

Torque converter as a system MIDDELMANN, Volker.. ( en linea) 2006. (consuktado el 25 de sptiembre de 2017.) disponible en: http://www.waghomswood.net.nz/Manuals/Vehicles/AllisonAutomatic/6torqueco nverter1.pdf

Las maquinas agricolas ORTIZ, Jaume Cavañate. y su aplicacion 2003. (consultado el 29 de septiembre de 2017) ISBN: 8484761177.

Torque Converter Clutch Optimization Robinette.: Improving Fuel Economy and Reducing Noise and Vibration 2011. (Consultado el 20 de Octubre de 2017).

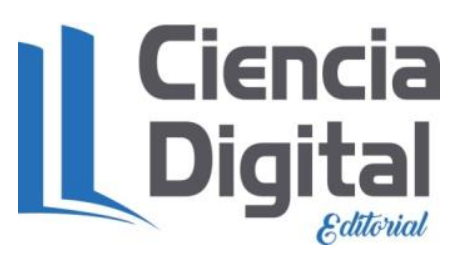




\section{Para citar el artículo indexado.}

Montúfar P., Bravo V., Castillo E., Sanchez E. \& Abacar E. . (2018). Análisis del diseño y material de un convertidor de par de una transmisión automática sobre el desempeño de torque y potencia de un automóvil liviano. Revista electrónica Ciencia Digital 2(3), 567586.

http://cienciadigital.org/revistacienciadigital2/index.php/CienciaDigital/article/view/176 $\underline{156}$

\section{Ciencia \\ LDigital}

El artículo que se publica es de exclusiva responsabilidad de los autores y no necesariamente reflejan el pensamiento de la Revista Ciencia Digital.

El articulo queda en propiedad de la revista y, por tanto, su publicación parcial y/o total en otro medio tiene que ser autorizado por el director de la Revista Ciencia Digital.
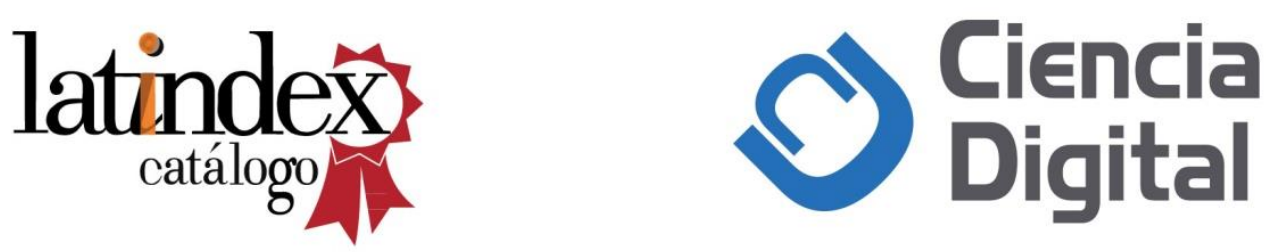